\title{
Paradoxical Relationship between Output and Input Regularity for the FitzHugh-Nagumo Model
}

\author{
Stuart Feerick, Jianfeng Feng, David Brown \\ Laboratory of Computational Neuroscience, The Babraham Institute, Cambridge, \\ CB2 4AT, UK.
}

\begin{abstract}
We examine the effects of changing the coefficient of variation (CV) of the inter-stimulus interval on the CV of the output interspike interval (ISI), using constant magnitude, supra-threshold pointprocess stimuli of the membrane potential variable in the FitzHughNagumo model. The coefficient of variation of the input is changed within the context of a displaced exponential distribution. We find that for some values of mean inter-stimulus interval, CV of ISI has an inverse relationship with input coefficient of variation, whereas for other mean stimulation rates, CV of ISI increases with input coefficient of variation. Over a wide range of input regularity CV of ISI is approximately constant, and is less than 0.4 .
\end{abstract}

\section{Introduction}

The FitzHugh-Nagumo (FHN) model is a simple model of neuronal excitability which retains much of the significant physiological behaviour of the Hodgkin-Huxley model. The behaviour of the excitable FHN model has been shown to be similar to that of living pacemakers [13], and its response to regular periodic forcing has been well researched $[1,4,12,7]$. Motivated by the regularity of pacemaker systems within the endocrine system [9], in this paper we investigate the behaviour of the FitzHugh-Nagumo model when subjected to stochastic point process input which is above the threshold required to cause an output spike from the FHN model. We find parameter regions of relative invariance of the output inter-spike interval (ISI) and other regions in which the behaviour is counter-intuitive, involving an inverse relationship between input and output variability. 


\section{Methods}

\section{$2.1 \quad$ Model}

Following Brown et al. [2], we use a version of the space clamped FHN model:

$$
\begin{gathered}
\frac{d v}{d t}=\gamma\left[-v(v-\alpha)\left(v-v_{\max }\right)-k_{1} w\right]+I_{\text {synaptic }} \\
\frac{d w}{d t}=\delta\left(k_{2} v-\beta w\right)
\end{gathered}
$$

For the simulations presented here $v_{\max }=1.0, \alpha=0.2, \gamma=200$, $\delta=0.9$. We also set $k_{1}=k_{2}=1.0 . \beta$ is varied between 1.0 and 5.0. $I_{\text {synaptic }}$ represents the applied stimulation.

We define a spike to have occurred when $v$ crosses a threshold (set here to 0.7) in an upward direction, provided a minimum interval (0.1 time units (TU)) has elapsed since the last threshold crossing.

\subsection{Applied stochastic stimulation}

The model uses instantaneous stochastic stimulations in $v$, arriving at randomly varying intervals $\Delta t_{i}$ consisting of a fixed refractory period of length $r$, followed by a period which follows a negative exponential distribution, mean $\tau$. Thus each $\Delta t_{i}$ follows a displaced negative exponential distribution, with displacement $\tau$ and overall mean $r+\tau$. We define:

$$
\begin{gathered}
p_{\text {stoch }}=\frac{\tau}{r+\tau} \\
\Delta t=\mathrm{E}\left(\Delta t_{i}\right)
\end{gathered}
$$

Thus, $p_{\text {stoch }}$ is the coefficient of variation of inter-stimulus interval, and $\Delta t$ is the mean inter-stimulus interval. $p_{\text {stoch }}=0$ corresponds to regular input, $p_{\text {stoch }}=1$ to Poissonian input. Perturbations are delivered with constant magnitude $\Delta v_{i}=0.35$, which is chosen to be super-threshold (i.e. $\Delta v_{i}$ is substantially greater than $\alpha$ ). 


\subsection{Simulations}

The simulation program was written in FORTRAN, using the NAG numerical libraries, and the equations were solved using a forward communication routine for integrating stiff systems. Simulations were run for 300 or 500 time units, after an initial transient of 5 time units which was discarded for the analyses presented here.

\section{Results}

\subsection{Changes in the mean inter-spike interval}

Example plots of $v$ are shown in Fig. 1 for three values of $p_{\text {stoch }}$ and two values of $\Delta t$. For $p_{\text {stoch }}=0.0$ and $\Delta t=0.1$, we observe many small perturbations in $v$, which are sub-threshold. Each of these corresponds to one input stimulation, and their frequency has the effect of constantly elevating $w$. This produces what has been termed a 'dynamic equilibrium' [1]; the perturbations accumulate so fast that the inhibitory variable, $w$, has insufficient time to decay between stimulations and effectively increases the threshold for spiking. The move away from the dynamic equilibrium toward more regular spiking can be seen in the bifurcation diagram (Fig. 2) which also shows that both $\Delta t=0.3,0.4$ are regions in which one output pulse is generated for every three input pulses.

We can obtain a more condensed representation of some of these results by plotting mean ISI against $p_{\text {stoch }}$, as given in Fig. 3. We observe that mean ISI is greatest for low $p_{\text {stoch }}$ and low $\Delta t(=0.1)$, and increasing either of these parameters allows the system to spike more frequently. A detailed description of how mean ISI changes as the input is randomly varied is presented in [6].

\subsection{Changes in output regularity}

We also calculated CV of ISI, and present graphs showing how it changes with $\Delta t$ and $p_{\text {stoch }}$ (see Fig. 4 ). For $\Delta t \geq 0.3$, we see output irregularity rises gently with $p_{\text {stoch. }}$. However for $\Delta t=0.1$, we observe the opposite relation.

Examination of the $v$ vs $t$ graphs for $\Delta t=0.1$ (Fig. 1, b) shows that the stimulus causing each output spike is often preceded by a 
(a)

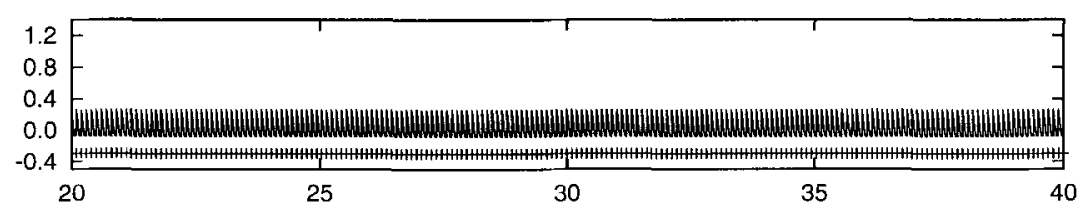

(b)

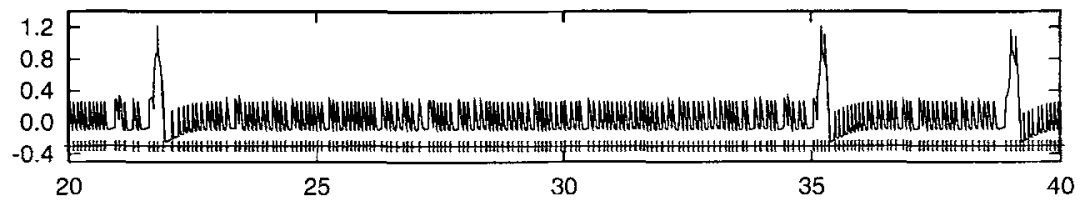

(c)

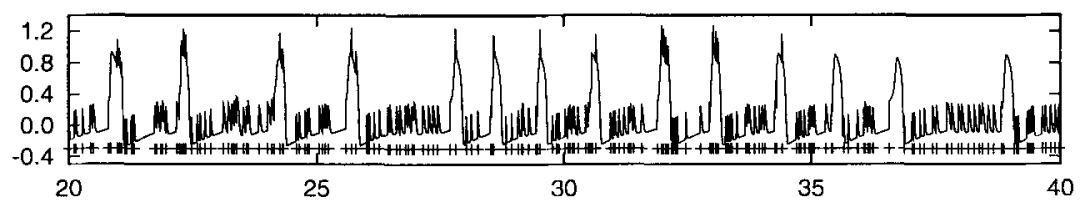

(d)

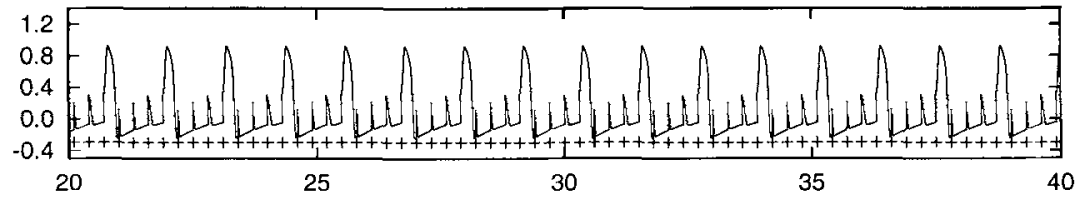

(e)

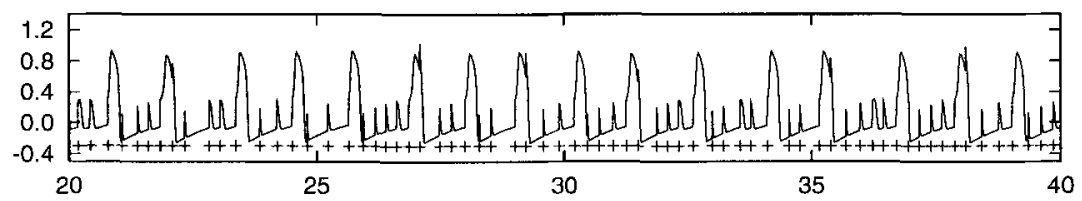

(f)

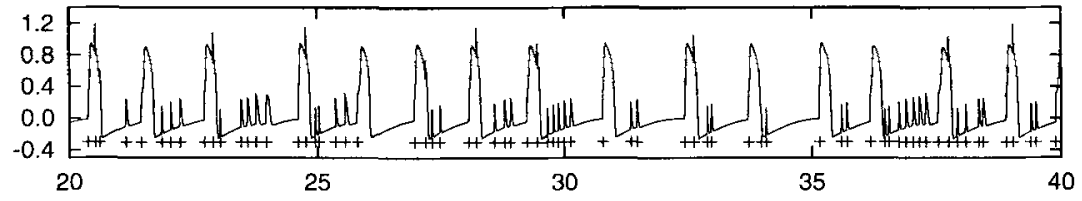

Fig. 1. Example simulations of the model showing $v$ vs. $t$ for $I_{\text {net }}=$ $0.0, v_{\max }=1.0, \alpha=0.20, \beta=1, \gamma=200, \delta=0.9, k_{1}=1.0, k_{2}=1.0$, and (a) $p_{\text {stoch }}=0.00, \Delta t=0.1$, (b) $p_{\text {stoch }}=0.35, \Delta t=0.1$, (c) $p_{\text {stoch }}=0.75, \Delta t=0.1$, (d) $p_{\text {stoch }}=0.00, \Delta t=0.3$, (e) $p_{\text {stoch }}=0.35$, $\Delta t=0.3$, and (f) $p_{\text {stoch }}=0.75, \Delta t=0.3$. Plus signs indicate input stimulations. 


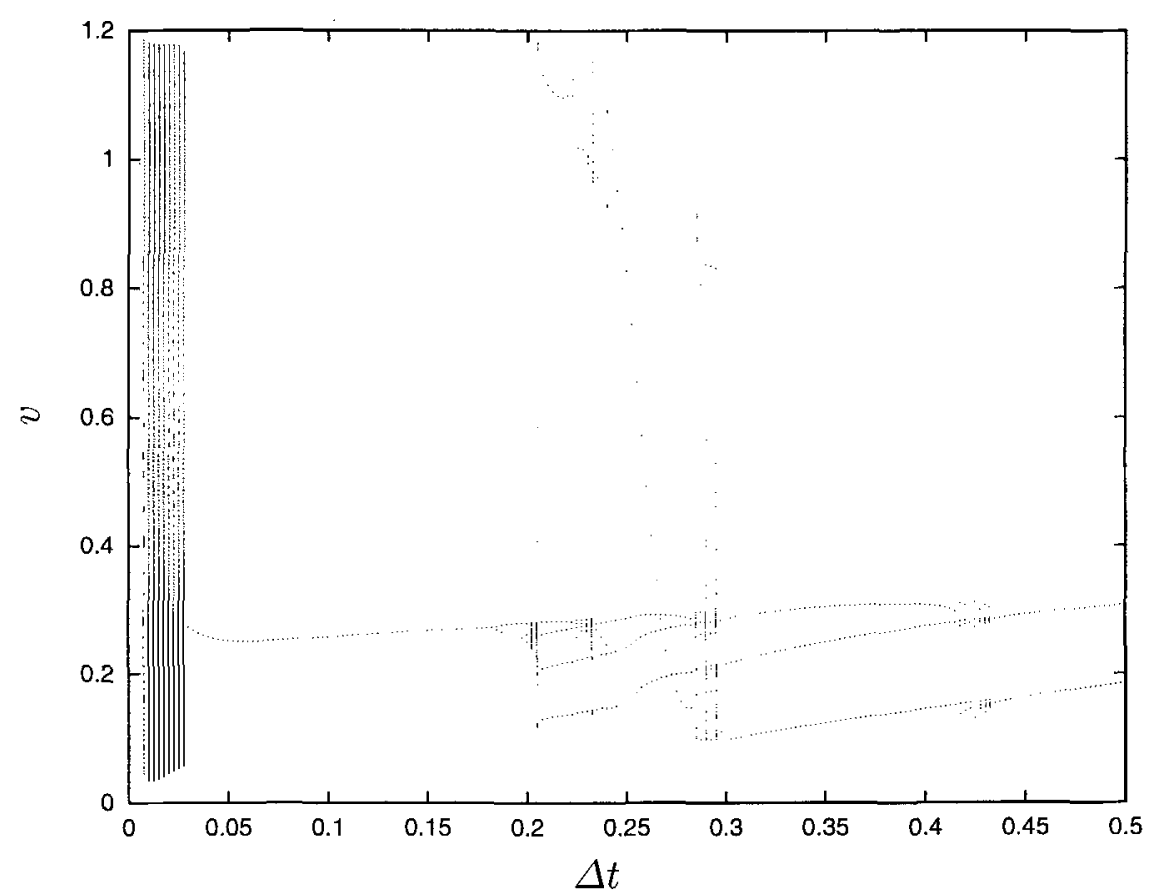

Fig. 2. Bifurcation diagram of $v$ as $\Delta t$ is varied. Other parameters as Fig. 1 Simulations were run for 30TU before being sampled for $30 \mathrm{TU}$

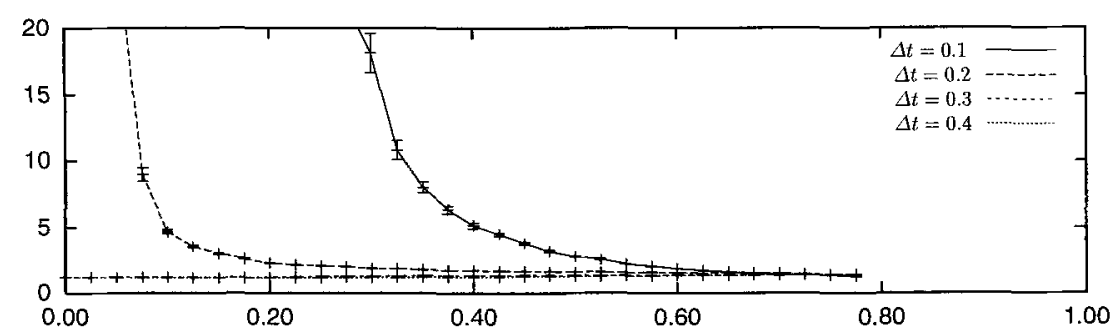

Fig. 3. Relationship between mean output inter-spike interval and $p_{\text {stoch }}$ for different $\Delta t$. Error bars show $\pm 1 \mathrm{SE}$. Other parameters as Fig. 1 


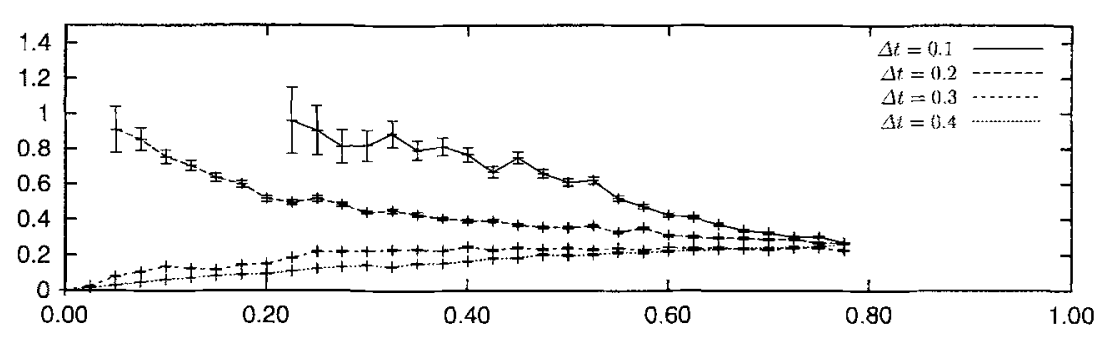

Fig. 4. Relationship between coefficient of variation of output interspike interval and $p_{\text {stoch }}$ for different $\Delta t$. Error bars show $\pm 1 \mathrm{SE}$. Other parameters as Fig. 1

relatively long period without any input stimulations. This allows the system to recover sufficiently to spike at the next stimulus. As the random potions of each inter-stimulus interval are independent negative exponentially distributed random variables, the occurrences of values greater than the threshold which permit a spike to occur, in effect, form a series of binomial trials. However, as firing becomes more frequent, the absolute refractory period between spikes establishes a regulatory effect, and the output is distributed as a fixed refractory period, followed by the outcome which is distributed as the first success in a sequence of binomial trials i.e. a geometric distribution. This explains the shape of the ISI histogram (Fig 5, $\Delta t=0.1$ ). The ISIs are skewed toward the minimum, and tail off approximately as a geometric distribution.

These simulation were repeated over a wide range of parameter space (not presented). Within the excitatory region, the results presented here are robust and the behaviours described are repeated.

\section{Discussion}

Previous studies have shown that the regularly stimulated FHN model exhibits complex relationship between input and output spike frequency $[1,13]$, and then that introducing and increasing a random component into the inter-stimulus interval and stimulus magnitude $[5,2]$ changes the relationship between mean ISI and interstimulus interval. In this paper, we extend these results by examin- 
(a)

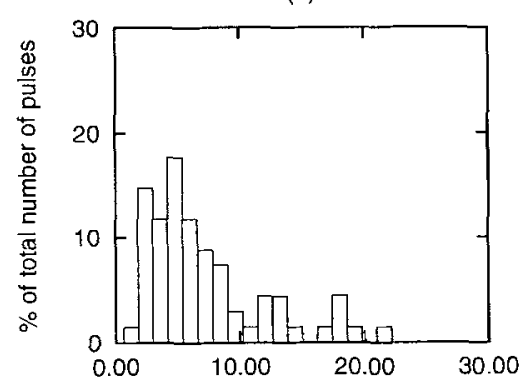

(c)

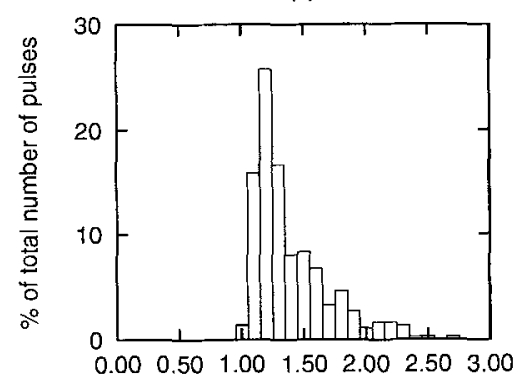

(b)

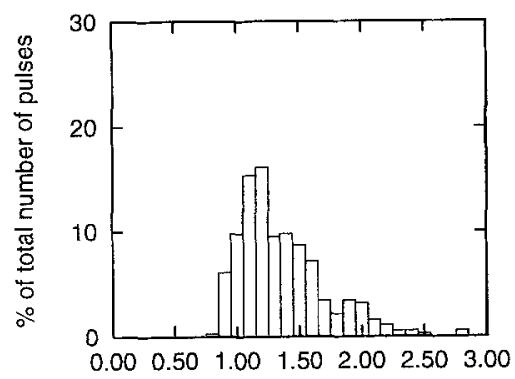

(d)

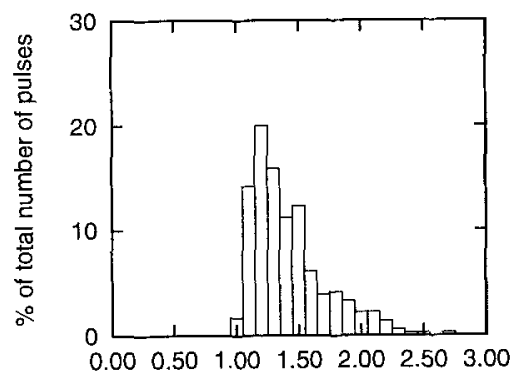

Fig. 5. Output inter-spike interval histograms for the model for: (a) $p_{\text {stoch }}=0.35, \Delta t=0.1$, (different scale) (b) $p_{\text {stoch }}=0.75, \Delta t=0.1$, (c) $p_{\text {stoch }}=0.35, \Delta t=0.3$, and (d) $p_{\text {stoch }}=0.75, \Delta t=0.3$. Other parameters as Fig. 1. Graphs show percentage of intervals falling into each bin based on sample sizes of at least 20,000.

ing the relationship between the regularity or otherwise of the input stimuli and the degree of regularity of the output spikes.

Perhaps the most striking result is the ability of a simple neuron to respond regularly to irregular input, over a wide region of parameter space. Away from the dynamic equilibrium, even as input tended toward a pure Poissonian distribution, the output remains regular, with $\mathrm{CV} \leq 0.4$. This result is repeatable within a wide region of parameter space, and is robust even when variation is introduced into the stimulation magnitude (results not presented). The source of the regularity is likely to be the refractory period, which is approximately 3 times the length of a spike, or between $2.5(\Delta t=0.4)$ and 10 $(\Delta t=0.1)$ times the period between input stimulations. Thus for a 
significant proportion of time, the system is in an effective refractory state; unexcitable even by the applied supra-threshold stimulations. A beat-skipping phenomenon [11] can be clearly observed in the histograms. The importance of this in contributing to the irregularity of the output is difficult to determine, though it undoubtedly increases output CV.

The original motivation for this work was further analysis of a descriptive network model of GnRH release [2], in which GnRH neurons interact with a reciprocally connected network of inhibitory GABA neurons. Output from the GnRH system is know to be pulsatile, and extremely regular. The present work suggests one way in which an inhibitory network can greatly increase the regularity of the output of a system. Experimental measures of pulse regularity are difficult, due to the wide distribution of the GnRH neurons throughout the pre-optic area of the brain which precludes accurate and prolonged measurement of the output of the system. However, inspection of available results $[14,3]$ suggests that pulse regularity changes significantly during the estrus cycle, and may provide important clues to changes in the pulse generator. The results presented are particularly significant in view of recent work which suggests that cultured GnRH neurons themselves may pulse spontaneously with great irregularity [8].

Neuroendocrinology offers many examples of neurons that are active in bursts [10], and our work in modelling these systems partially motivates the unusual, supra-threshold stimulations chosen in this paper. However, it is not impossible that a neuron may receive tightly synchronised inputs from connecting local neurons, which would be equivalent to a single, supra-threshold input. We intend to extend the input model to determine whether these results are repeatable for an input received as a burst of sub-threshold EPSPs within the physiological range.

\section{References}

1. David Brown, Jonathan P.A. Foweraker, and Robert W. Marrs. Dynamic equilibria and oscillations of a periodicially stimulated excitable system. Chaos, Solitons and Fractals, 5(3/4):359-369, 1995.

2. David Brown, Allan E. Herbison, Jane E. Robinson, Robert W. Marrs, and Gareth Leng. Modelling the luteinizing hormone-releasing hormone pulse generator. Neuroscience, 63:869-869, 1994. 
3. Neil P. Evans, Geoffrey E. Dahl, David T. Mauger, Vasantha Padmanabhan, and L.A. Thrun. Does estradiol induce the pre-ovulatory gonadotropin-releasing hormone surge in the ewe by inducing a progressive change in the model of operation of the GnRH neurosecretory system. Endocrinology, 136(12):5511-5519, 1995.

4. Robert FitzHugh. Impulses and physiological states in theoretical models of nerve membranes. Biophysical Journal, 1:445-466, 1961.

5. J.P.A. Foweraker and D. Brown. The effects of random variation in stimulation timing and magnitude on excitable and oscillatory forms of a luteinizing hormone pulse generator model. In Computation in Cellular and Molecular Biological Systems, pages 315-327. World Scientific, 1996.

6. J.P.A. Foweraker, D. Brown, and R.W. Marrs. Discrete-time simulation of the oscillatory and excitable forms of a FitzHugh-Nagumo model applied to the pulsatile release of luteinizing hormone releasing hormone. Chaos, 5(1):200-208, 1994.

7. L. Glass and M.C. Mackey. From Clocks to Chaos: The Rhythms of Life. Princeton University Press, 1988.

8. John D. Gordan, Barbara J. Attardi, and Donald W. Pfaff. Mathematical exploration of pulsality in cultured gonadotropin-releasing hormone neurons. Neuroendocrinology, 67:2-17, 1998.

9. Gareth Leng, editor. Pulsatility in neuroendocrine systems. CRC Press, Inc., Boca Raton, Florida, first edition, 1988.

10. Gareth Leng and David Brown. The origins and significance of pulsatility in hormone secretion from the pituitary. Journal of Neuroendocrinology, 9:493-513, 1997.

11. André Longtin. Stocastic resonance in neuron models. Journal of Statistical Physics, 70(1-2):309-327, 1993.

12. J. Nagumo, S. Arimoto, and S. Yoshizawa. An active pulse transmission line simulating nerve axon. Proceedings of the Institute of Radio Engineers, 50:20612071, 1962.

13. Shunsuke Sato and Shinji Doi. Response characteristics of the BVP neuron model to periodic pulse inputs. Mathematical Biosciences, 112:243-259, 1992.

14. T. Tanaka, Y. Mori, and K. Hosino. Hypothalamic GnRH pulse-generator activity during the estradiol-induced LH surge in ovariectomized goats. Neuroendocrinology, 56(5):641-645, 1992. 